Please do not remove this page

RMIT

UNIVERSITY

\title{
Quantitative analysis of rate-driven and due date-driven construction: production efficiency, supervision, and controllability in residential projects
}

Arashpour, Mehrdad; Wakefield, Ronald; Blismas, Nick; Abbasi, Babak

https://researchrepository.rmit.edu.au/esploro/outputs/9921862558201341/filesAndLinks?institution=61RMIT_INST\&index=null

Arashpour, M., Wakefield, R., Blismas, N., \& Abbasi, B. (2016). Quantitative analysis of rate-driven and due date-driven construction: production efficiency, supervision, and controllability in residential projects. Journal of Construction Engineering and Management, 142(1), 1-11.

https://doi.org/10.1061/(ASCE)CO.1943-7862.0001032

Document Version: Accepted Manuscript

Published Version: https://doi.org/10.1061/(ASCE)CO.1943-7862.0001032

Repository homepage: https://researchrepository.rmit.edu.au

(c) 2015 American Society of Civil Engineers.

Downloaded On 2023/04/26 21:25:55 +1000 
Thank you for downloading this document from the RMIT Research Repository.

The RMIT Research Repository is an open access database showcasing the research outputs of RMIT University researchers.

RMIT Research Repository: http://researchbank.rmit.edu.au/

\section{Citation:}

Arashpour, M, Wakefield, R, Blismas, N and Abbasi, B 2015, 'Quantitative analysis of rate-driven and due date-driven construction: production efficiency, supervision, and controllability in residential projects', Journal of Construction Engineering and Management, vol. 142, no. 1, pp. 05015012-1-05015012-11.

See this record in the RMIT Research Repository at:

https://researchbank.rmit.edu.au/view/rmit:33177

Version: Accepted Manuscript

Copyright Statement: (C) ASCE

Link to Published Version:

http://dx.doi.org/10.1061/(ASCE)C0.1943-7862.0001032

\section{PLEASE DO NOT REMOVE THIS PAGE}




\section{Quantitative analysis of rate-driven and due-date-driven \\ 2 construction: Production efficiency, supervision and \\ 3 controllability in residential projects}

4 Mehrdad Arashpour, M.ASCE ${ }^{1}$; Ron Wakefield, M.ASCE ${ }^{2}$; Nick Blismas ${ }^{3}$; and

5 BabakAbbasi ${ }^{4}$

\section{Abstract}

7 Concerns about production efficiency, quality and affordability in the residential construction

8 indicate there may be benefits in adopting alternative production control strategies to those

9 traditionally used. Reducing adverse effects of exogenous variability in demand and endogenous

10 variability in process are the ultimate goals of production control strategies. For residential

11 construction this means controlling the number of houses under construction and controlling the

12 start rate of new house constructions. The aim of this investigation is to compare and contrast the

13 outcomes of these two production management strategies. Production data of two volume house

14 builders in Victoria and Queensland, Australia, were collected. Tangible performance metrics of

15 builders were analyzed and compared using principles of the queuing theory. Then numerous

16 simulation experiments were designed and run in order to analyze different what-if scenarios in

17 the building environment. A special purpose simulation template was developed in order to

18 define a cap for production and limit the number of houses under construction based on actual

19 demand and available capacity. The findings reveal that rate-driven construction outperforms

20 due- date-driven construction in terms of three studied performance measures. This investigation

21 adopts an original and quantitative approach towards three production aspects of efficiency,

22 supervision and controllability. Therefore it contributes to the body-of-knowledge by developing

23 an in-depth insight into superior performance of the rate-driven control strategy with the 
24 intention of improving production output and long term sustainability of residential and other

25 sub-sectors of the construction industry.

26 CE Database subject headings: Engineering productivity; Lean construction; Modeling, Project

27 workflows; Work process simulation

28 Keywords: Computer simulation; Critical Path Method (CPM); Due-date-driven construction;

29 Efficiency; First-in-first-out (FIFO); Project management; Rate-driven construction; Residential

30 projects; Resource utilization; Queuing theory; Sensitivity analysis

$43{ }^{1}$ Lecturer, School of Property, Construction and Project Management, RMIT Univ., Melbourne, VIC, Australia; E-mail: 44 mehrdad.arashpour@rmit.edu.au

$45{ }^{2}$ Professor of Construction, Head of School of Property, Construction and Project Management, RMIT Univ., Melbourne, VIC, 46 Australia; E-mail: ron.wakefield@rmit.edu.au

$47{ }^{3}$ Professor, School of Property, Construction and Project Management, RMIT Univ., Melbourne, VIC, Australia; E-mail: 48 nick.blismas@rmit.edu.au

$49{ }^{4}$ Senior Lecturer, School of Mathematical and Geospatial Sciences, RMIT Univ., Melbourne, VIC, Australia; E-mail: 


\section{Introduction}

52 House building is an important sector of the construction industry that heavily relies on

53 subcontracting (Sawhney, Walsh et al. 2009). Evidence of shortage in supplying new housing

54 has been recognized by government and industry bodies. As an example in Australia, the 55 National Housing Supply Council (NHSC) and Housing Industry Association (HIA) estimate a 56 shortage of 466000 houses by 2020 .

57 In order to improve the situation, principles of production management have been borrowed from 58 manufacturing to enhance traditional methods of construction project management. For example, 59 resource driven scheduling or Critical Chain Project Management (CCPM), which is based on 60 the theory of constraints (Goldratt and Cox 2005), adds more accuracy to the Critical Path 61 Method (De la Garza and Kyunghwan 2009). Furthermore, lean construction (Sacks, Treckmann 62 et al. 2009) and even flow production (Bashford, Sawhney et al. 2003) are being increasingly 63 cited in the construction management literature as means of optimizing performance measures 64 such as lead time, profit, output/throughput $(\mathrm{TH})$, and service level.

65 The objective of workflow management or even flow production (EFP) is to ensure a smooth 66 work flow among several interacting trade contractors by means of reducing the variability in 67 their workload caused by fluctuating sales rates. In construction, using EFP was first proposed in 68 studying homebuilding projects in Phoenix Arizona (Bashford, Sawhney et al. 2003). They 69 reported on the superiority of EFP in terms of minimizing house completion times, workflow 70 variability, and management efforts. There are two distinguished strategies for system loading in 71 resource-constrained networks of production homebuilding, each with unique effects on 72 performance measures (Bashford, Walsh et al. 2005). 
73 The first, and traditional, method to manage system loading in the volume homebuilding is due 74 date driven in which builders schedule/push new jobs into the network so as to match the sales 75 rate. This strategy fails to maintain house completion times at a reasonable level and also creates 76 an unsustainable production flow especially during boom periods, when demand for building 77 new houses increases substantially and therefore resource constrained trades are not able keep up 78 (Lu and Lam 2008, Dalton, Wakefield et al. 2011). The second production control strategy is 79 called rate driven production. This strategy does not authorize a new construction start unless a 80 completed job leaves the network (Gurevich and Sacks 2014). Improvements made by a rate81 driven environment can be extended by controlling the number of houses under construction or 82 work-in-process (WIP). Maintaining a constant work-in-process (CONWIP) has positive effects 83 on tangible performance metrics of production homebuilders (Liu 2010, Arashpour and 84 Arashpour 2015). In fact, this workflow control protocol turns the network of trades into a closed 85 queuing system where unauthorized jobs from outside cannot enter. Despite the wealth of 86 research on alternative project planning and control strategies, further quantitative productivity 87 studies are required to evaluate the performance of such strategies and propose a continuous 88 productivity improvement process (Lucko, Alves et al. 2014). Furthermore, theoretical reasons 89 behind the superiority of these alternative strategies and the resultant practical issues need more 90 investigation (Gouett, Haas et al. 2011).

91 In order to bridge this gap, this paper quantitatively analyzes the performance of homebuilders 92 that use due-date driven strategy with those who take the alternative approach of rate-driven 93 production. Volume house building sector with its data rich environment is a suitable domain for 94 the purpose of this study. First, mathematical models of open and closed queues for individual 95 trades were built and analyzed. Since construction production networks are too complex to be 
solved analytically, in the next step simulation model of the whole trade contractor network was built and run in order to analyze and compare the collected data. Efficiency, coordination and supervisory requirements, and controllability are three areas under investigation in the current study. This paper suggests that rate driven production and its workflow leveling approach can improve both the economic sustainability and production efficiency of the homebuilding sector. Improvements in the house building sector from the use of rate driven production are likely to be generalizable to other sub-sectors of the construction industry due to their similarities.

\section{Production efficiency in construction projects}

Underperformance in the construction industry is a problem that is closely related to low productivity levels (Peña-Mora, Han et al. 2008, Skibniewski and Ghosh 2009, Arashpour and Arashpour 2010, Moselhi and Khan 2012).Using an appropriate production control strategy can improve performance metrics in different sectors of the industry, including residential construction. Due-date-driven and rate-driven construction are two production control strategies within the interconnected network of trade contractors in house building. Each strategy has unique effects on performance metrics.

Production data of two case studies were collected and utilized to validate the results of analytical and simulation modeling of house building networks driven by two control strategies of due-date-driven and rate-driven production. Selection of a major house building company with headquarters in Queensland and Victoria, Australia, enabled the authors to compare the performance of two similar building systems in terms of construction methods and processes. The major difference between Victorian and Queensland builders are the workflow control strategies they use. While Victorian builder uses a rate-driven production strategy, the mining boom in Queensland and the need to build more houses to accommodate workers, has 
119 encouraged the other builder to use a due-date-driven strategy where new jobs are

120 scheduled/pushed into the network so as to match the sales rate.

121 Production rate of the builders were computed by recording the number of houses started and

122 completed each month. Furthermore, the degree of workflow stability was calculated by

123 recording the standard deviation of time between completions. Several site observations and

124 documentary analysis were conducted in order to collect the required data. The notation and

125 symbols used for the modeling purpose are listed in the appendix of this manuscript.

126 One of the builders, coded as builder A in the current study, tries to match the production with 127 sales with the intention of meeting the agreed completion times. In other words, the level of 128 work-in-process $(W I P)$ or number houses under construction varies at times based on sales, 129 which is represented by the contractors' production output. In this way, WIP acts as a function 130 of output/throughput i.e. WIP $=f(T H)$. Throughput defines the rate at which jobs pass through 131 processes. This behavior closely represents due-date-driven production (Sacks and Goldin 2007, 132 Arashpour 2014). Fig. 1 shows a schematic illustration of the production workflow and control 133 in the due-date-driven construction.

Fig.1. Schematic flow of work and production control within the due-date-driven network

Fig. 1 illustrates due-date-driven construction, which is an open queuing network and jobs can

137 freely enter the network Based on forecast demand. As can be seen, new jobs are 138 scheduled/pushed into the network of trade contractors in a due-date-driven construction and the 139 main focus of production control system is on start and finish of assigned tasks to specialty 140 trades. 
141 However, the second builder, coded as builder B, starts a new house only after a completed

142 house exits production. In fact, the start rate to build new houses varies at times based on the 143 trade performance and output/throughput rate is a function of WIP inventory,i.e. $T H=$ $144 f(W I P)$. This strategy represents rate-driven production, where a new job is pulled into the 145 network upon the completion of one job by the very last processor. Fig. 2 shows the flow of 146 work in the rate-driven network.

147 Fig.2. Flow of work within the trade network in rate-driven production

Fig. 2 illustrates rate-driven production, which creates a closed queuing network with a maximum production bound. The stability of workflow in the rate-driven environment enables

151 the network of trade contractors to accommodate an expected level of demand easily. In order to 152 compare the efficiency of due-date-driven and rate-driven construction, processes of individual 153 trades were first modeled analytically and tangible performance measures were compared 154 quantitatively. Then, simulation models of the whole production network were built and run in 155 order to analyze different what-if scenarios in the real-life construction environment. Two 156 selected production builders use similar construction methods and processes. Behaviors of two 157 production networks in building 1000 detached suburban houses were analyzed and compared in 158 the two production networks.

\section{Open and closed queuing networks}

160 In this investigation, the construction processes in both due-date-driven and rate-driven 161 environments are subcontracted to up to 50 trade contractors. Construction methods and process 162 times are similar for specialized trades in the two house building networks and the output of a 163 trade is always required by the successors in order to perform their tasks. It is assumed that there 
164 is sufficient external demand for both production environments. The decision variable for the 165 due-date-driven builder is selecting the start rate of new houses. The trade network in this case 166 acts as an open queuing network where freely fluctuating WIP is observed and the rate of new 167 construction starts is controlled. Queues for houses waiting to be processed by trade contractors 168 can be modeled using queuing theory principles for first-in-first-out (FIFO) queues. According to 169 Kendall's notation (Kendall 1953), the most general form of queue for our case can be 170 represented by $G / G / 1$, in which a Generally distributed demand rate is processed by a trade in

171 a Generally distributed process time, one by one. This queue can realistically represent unsteady172 state construction processes because simplifying assumptions such as normal process times are 173 not required (Walsh, Sawhney et al. 2007).

174 Adopting a due-date-driven workflow, the expected number of jobs in the queue to be processed 175 by a trade $\left(W I P_{q}\right)$ can be modelled in a similar approach to Spearman and Zazanis (1992) as Eq. 176 (1).

$W I P=f(T H) \stackrel{\text { yields }}{\longrightarrow} W I P_{q}=\frac{T H}{1-T H}$

178 In Eq. 1,TH is the throughput rate of the trade. Understandably, $T H$ is equal to the rate of new 179 construction starts $\left(r_{a}\right)$ when there is not re-entrant flow or rework (Brodetskaia, Sacks et al. 180 2013). This assumption will be relaxed in the simulation modeling and analysis in the next 181 section. Since $N$ trades are interacting in the network, the total work-in-process inventory can be 182 approximated by Eq. (2).

$$
W I P_{\text {total }}=\sum_{1}^{q=N} W I P_{q}
$$


184 In order to model the rate-driven production strategy, a cap should be defined on the inventory of 185 work-in-process or number of houses under construction. In this scenario, random external 186 demand is not released to the trade network directly. For example, as suggested by González, 187 Alarcón et al. (2011), a work-in-process buffer can be placed in front of the first trade in order to 188 dampen the effects of demand variability. Consequently, the trade network acts as a closed 189 queuing network where WIP is closely controlled and the rate of new construction starts is 190 observed. In this production setting, throughput is a function of WIP and can be modeled in a 191 similar approach to Arashpour, Wakefield et al. (2013a) as Eq. (3).

$$
T H=f(W I P)=\frac{W I P}{W I P+N-1}
$$

193 In Eq. 3, $\mathrm{N}$ is the number of processors (trade contractors). It is worth mentioning that Eq. 2 and 194 Eq. 3 are simplified models that partially reflect the interaction of specialty trades and impacts on 195 the productivity of one another. More sophisticated models are required to capture the impact of 196 site congestion and the need for using on-demand resources such as tower cranes.

197 In order to make a fair comparison between efficiency of the due-date-driven and rate-driven 198 production, the required work-in-process inventory to achieve same levels of throughput rate 199 should be compared in both environments. Towards this aim, WIP is let to build up in the rate200 driven network and resultant throughput rate is computed using Eq. (3). Then, exactly same 201 throughput rates are inserted into the Eq. (1) and (2) in order to compute the required WIP 202 inventory in the due-date-driven production network. The surface chart in Fig.3 shows the work203 in-process inventory versus achieved throughput rate in the due-date-driven and rate-driven 204 production environments. 
205

206

207

208

209

210

211

212

213

214

215

216

217

218

219

220

221

\section{Simulation experimental framework}

223 The interconnected networks of trade contractors in the construction production are too complex

224 to be solved analytically (AbouRizk, Knowles et al. 2001, Halpin 2010). In such networks,

225 resource delays often result in formation of queues or waiting lines. Among other methodologies

226 to model construction queues, discrete event simulation (DES) is the most common used (Farid

227 and Koning 1994, Martinez 2010). In simulation of construction queuing systems, providing 
228 timely and proper input data with high temporal and spatial accuracy enhances the reliability of

229 decisions making based upon the simulation output (Akhavian and Behzadan 2014).

230 In this study, simulation experiments were designed and run in order to analyze real-life what-if 231 scenarios in the construction production. Stochastic variables of construction production were 232 analyzed using ARENA discrete event simulator. Performance metrics of the due-date-driven 233 and rate-driven production networks were measured by running the simulation experiments for a 234 long production period (16 months).

235 Nonlinear random demand rates and process times were accommodated into the model. In a 236 similar approach to Lee, Fung et al. (2013) and Chan, Yuen et al. (2015) demand rates and 237 process times were not fit to the theoretical statistical distributions such as exponential or 238 triangular, in order to increase the modeling precision. Instead, ARENA input analyzer was used 239 to divide the actual data into groups and calculate the proportion in each group. In this way, 240 accurate empirical distributions were formed for both demand and processes. On-site process 241 times of trade contractors were observed and recorded in order to ensure simulation models can 242 realistically represent operations of the two builders. Finally, models were verified and validated 243 by applying modifications recommended by the project and site managers.

244 Care should be taken in using empirical distributions in simulation of construction processes. 245 Although empirical distributions are more accurate than theoretical distributions because they are 246 built using the actual data, there are two major limitations in using them. First, a high quality 247 sampling with large numbers of data points is required to form an empirical distribution function 248 (EDF) without undesirable irregularities. Second, using the empirical distribution in simulation 249 of construction processes is more plausible when probability of occurring extreme events is low. 
250 This study, for example, uses empirical distributions to represent the repetitive operations in

251 residential construction as the probability of occurring a process with extremely short or long

252 durations lends itself to estimation and therefore realistic lower and upper bounds for EDF are

253 known.

254 The main challenge in modeling the rate-driven production was to set a desired cap for the 255 number of houses under construction. This production strategy cannot be precisely modeled 256 using ready-to-use constructs in most simulation systems (AbouRizk, Halpin et al. 2011). A 257 special purpose code in SIMAN simulation language was written that prevents the very first 258 trade contractor from starting a new house until a house completion by the very last trade 259 contractor. Towards this aim the cap for maximum number of jobs under construction was 260 defined using a variable named CONWIP (constant work-in-process). This variable is 261 decremented when a new job enters the construction network and incremented when a completed 262 job leaves the network. Authorization for starting a new job is only granted if the variable is 263 greater than zero. The simulation module for enforcing a rate-driven production is shown in 264 Fig.4.

Fig. 4. Defining the control protocol in the simulation model for rate-driven production

266 The simulation construct in Fig.4, ensures that the number of jobs in the construction network 267 never grows beyond the predefined cap. Interested readers can refer to Arashpour, Wakefield et 268 al. (2014a) for a more detailed treatment of the modeling approach. Due-date-driven production 269 network does not limit the number of houses under construction and new job starts are scheduled 270 (not authorized). The results of running simulation experiments for the due-date-driven and rate271 driven production are shown if Fig 5. 
274 Based on the simulation results in Fig. 5, the number of houses under construction for both

275 systems grows until two production networks are loaded up to their production capacity by

276 month 4. Then, rate-driven production manages to set the cap for the number of houses under

277 construction and WIP inventory never grows beyond this level. However, house completions in

278 the due-date-driven production fall behind the number of starts and WIP inventory continues to

279 grow, reaching a peak of 582 at the end of the simulation period.

280 This continuous ingrowth of WIP reflects congestion in the due-date-driven production network

281 and not surprisingly, this congestion inflates the house completion times. Based on the

282 simulation results, number of house completions in the house building network with a cap on

283 WIP level surpasses the network without this workflow control strategy. This fact is evident in

284 the surface chart illustrated in Fig. 6.

Fig. 6. Number of house completions (due-date-driven vs. rate-driven constrction)

287 Comparison of Fig. 5 and Fig. 6 reveals that although there are more houses under construction

288 in the due-date-driven network (Fig. 5), the output is less than the rate-driven network (Fig. 6).

289 This proves the fact that the rate-driven production is more efficient than due-date-driven

290 because a higher output level is achieved by having smaller levels of work-in-process inventory.

291 This is consistent with findings of Gurevich and Sacks (2014), indicating that defining a cap on

292 the work-in-process level can improve the efficiency in the construction production and enable

293 builders to operate their trade contractor network in a more cost-effective way. Furthermore, the

294 simulation results are in line with those obtained by analytical results in the previous section and 295 provide a measure of validation. 


\section{Supervisory and coordination requirements in the due-date-driven and rate-}

\section{7 driven construction}

298 There is a high level of variability in both process times and demand rates within the

299 construction and particularly the house building sector. Variability in the construction process is 300 caused by many factors such as accidents on worksites(De la Garza, Hancher et al. 2000), worker 301 fatigue and illness(Arashpour, Shabanikia et al. 2012), shortage in material supply(Castro302 Lacouture, Süer et al. 2009, Hwang, Park et al. 2012), and management-related issues (Cheng, 303 Huang et al. 2013). Furthermore, periods of boom and bust cause variable demand rates for the 304 construction of new houses.

305 When all the house building processes are subcontracted to trades, the builder is solely in charge 306 of sales, marketing and construction management. The major difficulty for the builder is to 307 manage the flow of work or 'hand-offs' among trade contractors(Walsh, Bashford et al. 2004).

308 This complex coordination task is undertaken by building supervisors. In the common practice in 309 the Australian house building, a supervisor usually coordinates construction processes of about 31015 houses. This makes supervisors a valuable and highly utilized resource in the production 311 house building (Dalton, Wakefield et al. 2011, Arashpour, Wakefield et al. 2015a). The objective 312 of this section of the study is to explore possible effects of due-date-driven and rate-driven 313 production on the supervisor workload.

\section{Analytical model}

315 Both due-date-driven and rate-driven production environments heavily rely on their building 316 supervisors in order to coordinate the flow of work within the network of trade contractors. In 317 order to develop a special model for comparing the supervisory conditions in the two production 318 environments, the annual target of building 1000 houses was converted it to 83 houses per month 319 and almost three houses per day. Due-date-driven production exposes the trade network to a 
320

321

322

323

324

325

326

327 having different levels of demand.

$P(d)=\sum_{1}^{n} \frac{e^{-\lambda} \lambda^{d}}{d !}$

In Eq. (4), $P(d)$ is the probability of having a given level of demand and $\lambda$ is the mean value for the demand rate. Understandably, the expected number of dayshaving a certain demand level can 331 be calculated using Eq. 5.

$E(d)=n \times p(d)$

In Eq. 5, $n$ is the duration of observation for our set objective. In the current analysis, there have

334 been 365 working days and 115 nonworking days during the study period (16 calendar months), 335 therefore $n=365$.

336 It is worth mentioning that sales rates and consequently job arrivals to the network are random.

337 After setting the throughput rate in the due-date-driven production, there exist periods when 338 supervisors are not busy. The probability of having no demand for constructing new houses 339 is $P(d=0)=e^{-3} \approx 5 \%$. Furthermore, the number of idle days for a supervisor can be 340 computed by Eq. 5 and is equal to $E(d=0)=365 e^{-3}=18$ days. 
341 During construction boom periods, it is also likely that sales rates are greater than the initial

342 estimation of the due-date-driven builder. The proportion of time when production is not able to

343 keep up with constraints in the capacity of trade contractors and supervisors can be calculated as,

$P(d>3)=1-P(d \leq 3)=1-\sum_{1}^{3} \frac{e^{-\lambda} \lambda^{d}}{d !}=1-\left(e^{-3}+\frac{3 e^{-3}}{1 !}+\frac{9 e^{-3}}{2 !}+\frac{27 e^{-3}}{3 !}\right) \approx 35 \%$

344 This indicates that over a long period of time (127 days in a working year) the due-date-driven 345 production experiences a slowdown, which is caused by trade contractor and supervisor 346 overload. This fact has also been illustrated in Fig.7. The shaded area shows the likelihood of 347 having greater demand than three houses per day.

Fig. 7. Probability distribution plot for the number of new construction starts (due-date-driven production)

It should be taken into consideration that sales and marketing strategies can play a major role in any residential development project. In the current investigation, such strategies are very similar as the builders are two regional branches of a major house building company in Australia. Understandably, a more detailed treatment of the sales/marketing strategies requires a more advanced modeling approach.

Overall, the results of this part of the study indicate that due-date-driven production faces difficulties to create a smooth workflow for building supervisors, who experience periods of idleness followed by periods of overload. In other words, supervision and coordination of construction processes is difficult to plan in the due-date-driven production.

In the next step, simulation experiments were designed in order to investigate the behavior of both due-date-driven and rate-driven production strategies with regard to building supervision and coordination. 


\section{Simulation experiments}

363 The house building processes in the rate-driven production environment were simulated.

364 Frequency statistics were collected in order to observe the daily status of supervisors (idle or 365 busy). In a similar approach to Arashpour, Wakefield et al. (2013b), a special purpose code in 366 SIMAN was developed to report on the resource status. The simulation models were run for 100 times in order to obtain the desired confidence interval of 99\%. Comparison of results for the due-date-driven and rate-driven production is shown in table 1.

\section{Table 1. Summary of frequency statistics (working status of building supervisors)}

As can be seen in the rate-driven production, there are only 20 observations when supervisors experience an excessive workload (supervision of more than 15 houses). More importantly, there was a far more balanced utilization level for the rate-driven than due-date-driven building supervisors, $54 \%$ versus $22 \%$ over the simulation period. It is worth mentioning that in real-life construction, resources are not dedicated to a single project and when their workflow is unstable, they might engage in another project. Rate-driven construction can stabilize the workflow in the network and therefore maximize availability of resources when they are needed.

Other resources in the construction network have a similar situation to what is shown in table 1. For example, roofing contractor had the highest long-term utilization level and was the bottleneck in the network. By defining a realistic cap for the production and limiting the number of houses under construction in the rate-driven network, roofing contractor and other trades were able to stabilize their workflow. This stability in rate-driven production is characterized by reduced average standard deviation of time between activity completions. Actual production data from the two cases such as process times were fed into the simulation model in order to compare 
385 production rate (average time between completions) and workflow stability (standard deviation

386 of time between activity completions) for 10 major house building processes.

387

388

389

390

391

392

\section{Controllability}

394 In order to compare the controllability of production for the two production control strategies, 395 two issues of practical implementation and robustness in dealing with control errors were 396 investigated.

\section{Practical implementation}

398 Throughput rate (the number of houses that pass through processes) is set based on the capacity 399 estimations, which can only be done based on detailed information about work efficiency, 400 construction process times, rework and interruptions in the worksites (Sawhney, Walsh et al. 401 2009). Based on an estimation of the true capacity of the trade network in the due-date-driven 402 house building, throughput is set to the rate of new construction starts $\left(r_{a}\right)$, assuming that there is 403 no re-entrant flow or rework involved. Upper bound of the throughput rate is limited by the 404 performance of the trade network and is beyond the builder's control. In this way the function of 405 THcan be stated as: 


$$
T H=\left\{\begin{array}{lr}
\text { Start rate }\left(r_{a}\right) & \text { if }: r_{a}<\text { capacity } \\
\text { Capacity } & \text { Otherwise }
\end{array}\right.
$$

406 Commonly, an overestimation of the capacity leads the due-date-driven production strategy to

407 allow for excessive number of construction starts by the trade network and consequently the 408 number of houses under construction will grow rapidly. This is particularly true during 409 construction boom periods when a higher numbers of house completions than normal are desired.

410 This fact makes the implementation of the due-date-driven production strategy very risky.

411 Another issue regarding the practical implementation of due-date-driven is the utilization rate of

412 trade contractors. Results from running the simulation experiments showed that 50 trade

413 contractors in the due-date-driven production network experience high levels of variations in the

414 flow of work and frequent periods of idleness. This variable workload is difficult and expensive 415 for the trade contractors to accommodate (Bashford, Sawhney et al. 2003, Arashpour and 416 Arashpour 2012). As can be seen in Fig.8, utilization rates (the proportion of time that a trade is 417 busy) fluctuate between 62 and 99 per cent in the due-date-driven homebuilding network.

418 Fig.8. Utilization rates for 50 trade contractors (Simulation results for due-date-driven and rate419 driven production)

420

421 As it is evident in Fig. 8, in the rate-driven house building network, where the number of houses 422 under construction is bounded, trade contractors are more evenly utilized. Here the rates only 423 vary between 80 and 99 per cent and therefore trade contractors can be confident that they will 424 have a continuous flow of work.

425 While achieving a true capacity estimations and balanced utilization of trade contractors is 426 difficult in the due-date-driven production environment, rate-driven production directly observes 
427 the number of houses under construction. This is consistent with findings ofBallard (2000) and

428 Koskela, Sacks et al. (2012) indicating that the rate-driven production is a more practical strategy

429 in order to control the flow of work and utilization within the trade networks.

\section{Model validity}

431 Throughout this paper, analytical models have been developed to model system performance

432 measures in the residential construction context. Despite the use of simplifying

433 assumptions/approximations, the conjecture is that developed models are still reasonable

434 representations of the real system. Cross-comparison of simulation and analytical results has

435 been used to support this conjecture throughout the paper.

436 Furthermore, simulation results were validated using a systematic approach similar to Cates

437 (2004) and Mielczarek (2013). In the first step, case study participants were briefed about the

438 methodology used to develop the model and the way actual data were used to determine

439 empirical distributions. A total of 25 professionals were approached, six construction engineers,

440 four architects, 12 subcontractors, and three construction managers. Comments and final

441 agreement of case study participants on the model resulted in a high level of face validity.

442 Simulation models were expected to achieve a high face validity because in this study, existing

443 construction production networks were modelled. Furthermore, since empirical distributions

444 have been primarily used in this study, project participants were able to recognize data and relate

445 them to actual production. As a result, participants were inclined to accept the modelling

446 assumptions and simulation logic as the model reasonably represented the existing production

447 network and yielded the same output as the actual system. Achieving a high face validity is a

448 very important step in checking the model credibility as project participants have the deepest

449 understanding and insight into the ongoing production scenarios (Fellows and Liu 2008). 
450 In the second step and in order to validate the process of input-output transformation in the

451 simulation model, regular daily production processes of the two cases were modeled and run 500

452 times. Average time between activity completions (mean value and standard deviation) was

453 checked against the actual data collected from February to October 2014. The real-world

454 production data and simulation results were almost identical, with errors within the range of

$455 \quad 0.3 \%$. Table 3 shows the comparison between the results of the discrete event simulation model 456 and observed completion intervals.

\section{Table 3. Validation of simulation results against actual completion intervals}

458

459 In the third and final step, a sensitivity analysis was conducted by slightly manipulating input 460 variables to the model and this caused no extreme variation in model outputs. As an example, the 461 start rate of new houses were increased in the simulation model (1\% growth in $\left.r_{a}\right)$. This caused 462 only $0.5 \%$ increase in the number of houses under construction (WIP), which is acceptable as 463 WIP is also dependent to other variables such as house completion times $(C T)$. By completion of 464 the three steps of model validation process, results of simulation were considered valid and 465 reasonably robust.

\section{Robustness}

467 In order to compare the robustness of the two production strategies in dealing with control errors,

468 the optimization problem of balancing the cost of excessive number of houses under construction 469 and the cost of missed sales opportunities was considered. An excessive work-in-process results 470 in direct and indirect costs such as on-going site establishment costs and overheads. The 471 optimization problem, attempts to maximize the builder profit by finding a balance between 
472 throughput and work-in-process levels. The profit function of the production can be formulated 473 as Eq. (6).

474

Profit $=\alpha_{1} \times T H-\alpha_{2} \times W I P$

475 In Eq. (6), $\alpha_{1}$ is the builder profit for signing a new house contract and $\alpha_{2}$ is the total cost

476 associated with an uncompleted house in the builder's production network such as on-going

477 worksite establishment costs and late completion penalties. Let the profit of a new construction

478 start to be much higher than costs of having an uncompleted house in the production

479 network $\left(\alpha_{1}=1000 \alpha_{2}\right)$. That is, any house going through the homebuilding processes has the

480 potential to generate 1000 units of profit and to incur only one unit of cost. Understandably, 481 assuming a high profit/cost ratio, changes cost efficiencies in favor of the due-date-driven 482 production. This encourages the start of more new houses, even if production resources for on 483 time completion of those houses in the specialty trade network are currently unavailable. In the 484 rate-driven production, however, the main idea is to start a certain number of houses that can be 485 completed on time based on the available capacity (production resources).

486 Values of TH and WIP were computed using Eq. 1 and 3 for the due-date-driven (open queuing 487 network) and rate-driven (closed queuing network) respectively. The internal optimization tool in 488 MS Excel was used to find the optimal values of $T H$ and WIP that maximizes the builder's 489 profit. Rate-driven production profit can reach a peak of \$13.6 M by bounding the work-in490 process level to 240 houses. For the due-date-driven production, the best rate of jobs passing 491 through the processes is three houses per day, yielding a profit of $\$ 13.4 \mathrm{M}$. Therefore, the 492 maximum profit level for the rate-driven production is slightly (around two per cent) more than 
493 the due-date-driven production. Table 4 shows profit values for different $T H$ and $W I P$ levels in

494 the two production environments.

495

496

497

498

499

500

501

502

503

504

505

506

507

508

\section{Conclusions}

510 Previous research has documented the implications of rate-driven production in construction

511 (Bashford, Sawhney et al. 2003, Koskela, Sacks et al. 2012). The contribution of this paper to the

512 body of knowledge can be summarized to two parts. Firstly, the proposed control protocol for

513 maintaining a constant number of houses under construction limits the number of jobs to the

514 capacity of the specialty trade network. This flexible approach receives available capacity signal

515 from the production network and pulls new jobs into it. It has also the flexibility of pulling from 
516 the bottleneck and start of a new house can be authorized when bottleneck finishes the work on

517 an in-process job. This original perspective makes the rate-driven method flexible and applicable

518 to many construction networks even those using more complex production strategies than the

519 traditional subcontracting system. A special purpose simulation model has been developed in this

520 research to implement the rate-driven production in the house building environment. Secondly,

521 this paper addresses a gap in the construction research where quantitative performance

522 assessment of alternative project planning and control strategies are required. This investigation

523 analyzes the theoretical reasons behind the superiority of rate-driven production and the resultant

524 practical issues in this environment.

525 Based on the results, adopting the rate-driven production control strategy along with maintaining

526 a constant level of work-in-process can significantly improve tangible performance metrics in

527 volume homebuilding. The findings extend those of Sacks and Goldin (2007) and Koskela

528 (2000), confirming that direct control of the work-in-process inventory is more feasible than

529 indirect control of throughput and capacity estimations in the due-date-driven environment.

530 Furthermore, results of analytical models and simulation experiments produced several key

531 observations about the superiority of rate-driven production in the real world construction, such

532 as robustness against errors in determining the optimum number of houses under construction. In

533 fact, optimism in estimating production capacity and the desire to yield as much throughput as

534 possible to maximize profit are making due-date-driven production prone to errors in the control

535 parameters. That is, overestimating the capacity of the trade contractors' network results in more

536 construction starts and can lead to a loss of money and therefore cash flow problems for the 537 builders. 
538 The research reported in this paper builds up on the current body of knowledge by developing an 539 in-depth insight into the rate-driven and due-date-driven production control strategies.

540 Particularly, this study confirms and extends findings of Gurevich and Sacks (2014) and

541 Arashpour, Wakefield et al. (2014b) by adopting an original approach towards three performance

542 metrics of production efficiency, supervision and controllability.

543 Furthermore, residential builders can control their production network in a more cost-effective

544 way and improve the performance by adopting a rate-driven strategy. This can address the 545 problem of shortage in housing supply was the motivation for conducting this research. The

546 authors are currently working on the issue of market demand in order to find optimal ways of

547 buffering against demand variability in the rate-driven construction production. Future research 548 should include more stochastic variables in analyzing effects of rate-driven construction on 549 performance, productivity and process flexibility.

\section{Acknowledgements}

551 The project is partly funded by HDRPG-2014 Grant from the School of Graduate Research at 552 RMIT University. The authors would like to express their appreciation to three anonymous 553 reviewers in the Journal of Construction Engineering and Management whose constructive 554 comments have improved quality of this work substantially.

\section{Appendix. Notation and symbols}

556 WIP Work-in-process (Number of houses under construction)

$557 \quad$ TH Throughput of the production network

$558 \quad C T \quad$ Completion time

$559 \quad E($.$) \quad Expected value$ 


\begin{tabular}{|c|c|c|}
\hline 560 & $P()$. & Probability of \\
\hline 61 & $f()$. & Function of \\
\hline 62 & $r_{a}$ & Rate of starting new houses \\
\hline 63 & $\mu$ & Average time between completions \\
\hline 64 & $\sigma$ & Standard deviation of time between completions \\
\hline 65 & CONWIP & Constant work-in-process (Number of houses under construction capped) \\
\hline 56 & \multicolumn{2}{|c|}{ References } \\
\hline $\begin{array}{l}567 \\
568 \\
569\end{array}$ & \multicolumn{2}{|c|}{$\begin{array}{l}\text { AbouRizk, S., D. Halpin, Y. Moha } \\
\text { for improving construction en } \\
\text { Management 137(10): } 843 .\end{array}$} \\
\hline 570 & \multicolumn{2}{|c|}{ AbouRizk, S., P. Knowles and U. Herman (2001). "Estimating Labor Production Rate for Industrial } \\
\hline 71 & Constr & ion Activities." Journal of construction engineering and management 127(6): 502-511. \\
\hline $\begin{array}{l}572 \\
573\end{array}$ & $\begin{array}{r}\text { Akhavian, } \mathrm{I} \\
\text { simula }\end{array}$ & $\begin{array}{l}\text { and A. H. Behzadan (2014). "Evaluation of queuing systems for knowledge-based } \\
\text { n of construction processes." Automation in Construction 47: 37-49. }\end{array}$ \\
\hline $\begin{array}{l}574 \\
575\end{array}$ & $\begin{array}{l}\text { Arashpour, } \\
\text { flexibi }\end{array}$ & (2014). FULFIL: production control system for managing workflow, quality and \\
\hline 576 & Arashpour, & and M. Arashpour (2010). Gaining the Best Value from HR in Construction Companies. \\
\hline 577 & Procee & gs of the 6th European Conference on Management Leadership and Governance, \\
\hline 578 & Acadel & Conferences and Publishing International Limited, London, UK. \\
\hline 579 & Arashpour, & and M. Arashpour (2012). A collaborative perspective in green construction risk \\
\hline 80 & manag & ent. 37th Annual Conference Of The Australasian Universities Building Educators \\
\hline 581 & Associ & on (AUBEA), UTS Publishing/University of New South Wales. \\
\hline 582 & Arashpour, & and M. Arashpour (2015). "Analysis of Workflow Variability and Its Impacts on \\
\hline 583 & Produc & ity and Performance in Construction of Multistory Buildings." Journal of Management in \\
\hline 584 & Engine & ng: 040150061. \\
\hline 585 & Arashpour, & , M. Shabanikia and M. Arashpour (2012). "Valuing the contribution of knowledge- \\
\hline 586 & oriente & vorkers to projects: a merit based approach in the construction industry." Australasian \\
\hline 587 & Journa & f Construction Economics and Building 12(4): 1- 12. \\
\hline 588 & Arashpour, & , R. Wakefield and N. Blismas (2013b). Improving construction productivity: implications \\
\hline 589 & of ever & ow production principles. CIB World Building Congress 2013: Construction and Society, \\
\hline 590 & Queen & nd University of Technology. \\
\hline & shpour, & , R. Wakefield, N. Blismas and E. W. M. Lee (2013a). "A new approach for modelling \\
\hline 593 & $\begin{array}{l}\text { varlabl } \\
\text { Buildir }\end{array}$ & $\begin{array}{l}\text { in residential construction projects." Australasian Journal of Construction Economics and } \\
\text { 13(2): 83-92. }\end{array}$ \\
\hline 594 & Arashpour, & , R. Wakefield, N. Blismas and E. W. M. Lee (2014a). "Analysis of disruptions caused by \\
\hline 595 & constru & n field rework on productivity in residential projects." Journal of Construction \\
\hline 596 & Engine & ng and Management 140(2): 1-12. \\
\hline 597 & Arashpour, & , R. Wakefield, N. Blismas and E. W. M. Lee (2014b). "Framework for improving \\
\hline & workf & ability: Deployment of optimized capacity buffers in a synchronized construction \\
\hline & & 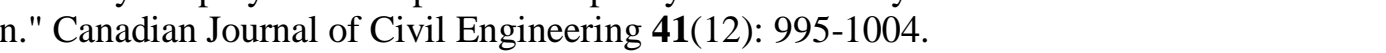 \\
\hline
\end{tabular}


Arashpour, M., R. Wakefield, N. Blismas and T. Maqsood (2015b). "Autonomous production tracking for augmenting output in off-site construction." Automation in Construction 53(0): 13-21.

Arashpour, M., R. Wakefield, N. Blismas and J. Minas (2015a). "Optimization of process integration and multi-skilled resource utilization in off-site construction." Automation in Construction 50(1): 72-80.

Ballard, H. G. (2000). The last planner system of production control, PhD dissertation, University of Birmingham.

Bashford, H. H., A. Sawhney, K. D. Walsh and K. Kot (2003). "Implications of even flow production methodology for U.S. housing industry." Journal of construction engineering and management 129(3): 330-337.

Bashford, H. H., K. D. Walsh and A. Sawhney (2005). "Production system loading-cycle time relationship in residential construction." Journal of construction engineering and management 131(1): 15-22.

Brodetskaia, I., R. Sacks and A. Shapira (2013). "Stabilizing Production Flow of Interior and Finishing Works with Reentrant Flow in Building Construction." Journal of Construction Engineering and Management 139(6): 665-674.

Castro-Lacouture, D., G. A. Süer, J. Gonzalez-Joaqui and J. K. Yates (2009). "Construction project scheduling with time, cost, and material restrictions using fuzzy mathematical models and critical path method." Journal of Construction Engineering and Management 135(10): 1096-1104.

Cates, G. R. (2004). Improving project management with simulation and completion distribution functions, PhD dissertation, University of Central Florida Orlando, Florida.

Chan, R. W. K., J. K. K. Yuen, E. W. M. Lee and M. Arashpour (2015). "Application of NonlinearAutoregressive-Exogenous model to predict the hysteretic behaviour of passive control systems." Engineering Structures 85(1): 1-10.

Cheng, M.-Y., C.-C. Huang and A. F. V. Roy (2013). "Predicting Project Success in Construction using an Evolutionary Gaussian Process Inference Model." Journal of Civil Engineering and Management 19(sup1): S202-S211.

Dalton, T., R. Wakefield and R. Horne (2011). "Australian suburban house building: industry organisation, practices and constraints." Australian Housing and Urban Research Institute Positioning Paper Series: 1-56.

De la Garza, J., D. Hancher and L. Decker (2000). "Analysis of Safety Indicators in Construction." Journal of Construction Engineering and Management 124(4): 312-314.

De la Garza, J. and K. Kyunghwan (2009). Application of the Resource-Constrained Critical Path Method to Multiple Calendars and Progressed Schedules. Construction Research Congress 2009, American Society of Civil Engineers: 916-925.

Farid, F. and T. Koning (1994). "Simulation Verifies Queuing Program for Selecting Loader-Truck Fleets." Journal of Construction Engineering and Management 120(2): 386-404.

Fellows, R. F. and A. M. M. Liu (2008). Research methods for construction, Wiley-Blackwell.

Goldratt, E. M. and J. Cox (2005). "A Process of Ongoing Improvement." Journal of Manufacturing Technology Management 16(3): 302.

González, V., L. Alarcón, S. Maturana and J. Bustamante (2011). "Site Management of Work-in-Process Buffers to Enhance Project Performance Using the Reliable Commitment Model: Case Study." Journal of construction engineering and management 137(9): 707-715.

Gouett, M., C. Haas, P. Goodrum and C. Caldas (2011). "Activity Analysis for Direct-Work Rate Improvement in Construction." Journal of Construction Engineering and Management 137(12): 1117-1124.

Gurevich, U. and R. Sacks (2014). "Examination of the effects of a KanBIM production control system on subcontractors' task selections in interior works." Automation in Construction 37: 81-87.

Halbach, E. and A. Halme (2013). "Job planning and supervisory control for automated earthmoving using 3D graphical tools." Automation in Construction 32(0): 145-160.

Halpin, D. W. (2010). Construction management, Wiley. com.

Hopp, W. J. and M. L. Spearman (2011). Factory physics, 3rd edn, Waveland. 
Hwang, S., M. Park, H. Lee, S. Lee and H. Kim (2012). "Dynamic Feasibility Analysis of the Housing Supply Strategies in a Recession: Korean Housing Market." Journal of Construction Engineering and Management 139(2): 148-160.

Kendall, D. G. (1953). "Stochastic processes occurring in the theory of queues and their analysis by the method of the imbedded Markov chain." The Annals of Mathematical Statistics: 338-354.

Koskela, L. (2000). An exploration towards a production theory and its application to construction, PhD dissertation, Helsinki University of Technology, VTT Technical Research Centre of Finland.

Koskela, L., R. Sacks and J. Rooke (2012). A brief history of the concept of waste in production. 20th Conference of the International Group for Lean Construction, IGLC 2012, San Diego, CA.

Lee, E. W. M., I. W. H. Fung, V. W. Y. Tam and M. Arashpour (2013). "A fully autonomous kernelbased online learning neural network model and its application to building cooling load prediction." Soft Computing: 1-16.

Liu, J. J. (2010). Inventory control through a CONWIP pull production system. PhD Dissertation, Massachusetts Institute of Technology.

Lu, M. and H. C. Lam (2008). "Critical path scheduling under resource calendar constraints." Journal of Construction Engineering and Management 134(1): 25-31.

Lucko, G., T. D. C. L. Alves and V. L. Angelim (2014). "Challenges and opportunities for productivity improvement studies in linear, repetitive, and location-based scheduling." Construction Management and Economics 32(6): 575-594.

Martinez, J. C. (2010). "Methodology for Conducting Discrete-Event Simulation Studies in Construction Engineering and Management." Journal of Construction Engineering and Management-Asce 136(1): 3-16.

Mielczarek, B. (2013). "Simulation modelling for contracting hospital emergency services at the regional level." European Journal of Operational Research(0).

Moselhi, O. and Z. Khan (2012). "Significance ranking of parameters impacting construction labour productivity." Construction Innovation 12(3): 272-296.

Palaniappan, S., A. Sawhney, H. H. Bashford and K. D. Walsh (2007). Special purpose simulation template for workflow analysis in construction. Winter Simulation Conference, Washington, DC.

Peña-Mora, F., S. Han, S. Lee and M. Park (2008). "Strategic-Operational Construction Management: Hybrid System Dynamics and Discrete Event Approach." Journal of Construction Engineering and Management 134(9): 701-710.

Sacks, R. and M. Goldin (2007). "Lean Management Model for Construction of High-Rise Apartment Buildings." Journal of construction engineering and management 133(5): 374-384.

Sacks, R., M. Treckmann and O. Rozenfeld (2009). "Visualization of Work Flow to Support Lean Construction." Journal of construction engineering and management 135(12): 1307-1315.

Sawhney, A., K. D. Walsh, H. H. Bashford and S. Palaniappan (2009). "Impact of inspected buffers on production parameters of construction processes." Journal of construction engineering and management 135(4): 319-329.

Skibniewski, M. J. and S. Ghosh (2009). "Determination of key performance indicators with enterprise resource planning systems in engineering construction firms." Journal of construction engineering and management 135(10): 965-978.

Spearman, M. L. and M. A. Zazanis (1992). "Push and Pull Production Systems: Issues and Comparisons." Operations Research 40(3): 521-532.

Walsh, K. D., H. H. Bashford, A. Sawhney and A. Witjakso (2004). "Cost of risk transfer: Pricing agreements in residential supply chains." Journal of Architectural Engineering 10(3): 112-118.

Walsh, K. D., A. Sawhney and H. H. Bashford (2007). "Production equations for unsteady-state construction processes." Journal of construction engineering and management 133(3): 254-261. 